\title{
A Deep Learning Model for Predicting Tumor Suppressor Genes and Oncogenes from PDB Structure
}

\author{
Amirhossein Tavanaei, Nishanth Anandanadarajah, Anthony Maida, and Rasiah Loganantharaj \\ The Center for Advanced Computer Studies \\ The School of Computing and Informatics \\ University of Louisiana at Lafayette \\ Lafayette, LA 70503, USA \\ \{tavanaei,nishanth,raja\}@louisiana.edu
}

\begin{abstract}
While cancer is a heterogeneous complex of distinct diseases, the common underlying mechanism for uncontrolled tumor growth is due to mutations in protooncogenes and the loss of the regulatory function of tumor suppression genes. In this paper we propose a novel deep learning model for predicting tumor suppression genes (TSGs) and proto-oncogenes (OGs) from their Protein Data Bank (PDB) three dimensional structures. Specifically, we develop a convolutional neural network (CNN) to classify the feature map sets extracted from the tertiary protein structures. Each feature map set represents particular biological features associated with the atomic coordinates appearing on the outer surface of protein's three dimensional structure. The experimental results on the collected dataset for classifying TSGs and OGs demonstrate promising performance with $82.57 \%$ accuracy and 0.89 area under ROC curve. The initial success of the proposed model warrants further study to develop a comprehensive model to identify the cancer driver genes or events using the principle cancer genes (TSG and OG).
\end{abstract}

Keywords: cancer, tumor suppressor gene, oncogene, deep learning, convolutional neural network

\section{Introduction}

Common themes (https://www. cancer.gov/about-cancer) among many different types of cancer at the molecular level include (1) mutations in proto-oncogenes that alter the function of the regular cell cycle to uncontrollable cell division, (2) mutations in cancer suppressor genes that alter their cell regulatory mechanism, and (3) mutations in DNA-repair genes that cause further mutations in cells instead of repairing them. Traditional machine learning algorithms such as decision trees, random forests (RF), artificial neural networks (ANN), support vector machines (SVM) have been successfully applied to build predictive models for various aspects related to cancer including prognosis of cancer, classification of cancer types from data sources such as clinical data, SNP's, gene expressions [1, 2, 3, 4, 5]. Recently, deep learning [6, 7] has shown remarkable performances for predicting the specificity of DNA and mRNA binding sites [8], functional classification [9], protein folding pattern [10], and for cancer categorization [11, 12]. Automatic detection and prediction of the either oncogenes or cancer suppression genes from their three dimensional features is a big step in discovering their structural characteristics to improve the state-of-the-art in making a dent in cancer treatments. To our knowledge there is no or few works done in applying machine learning in identifying oncogenes or cancer suppression genes from the three dimensional structures. 

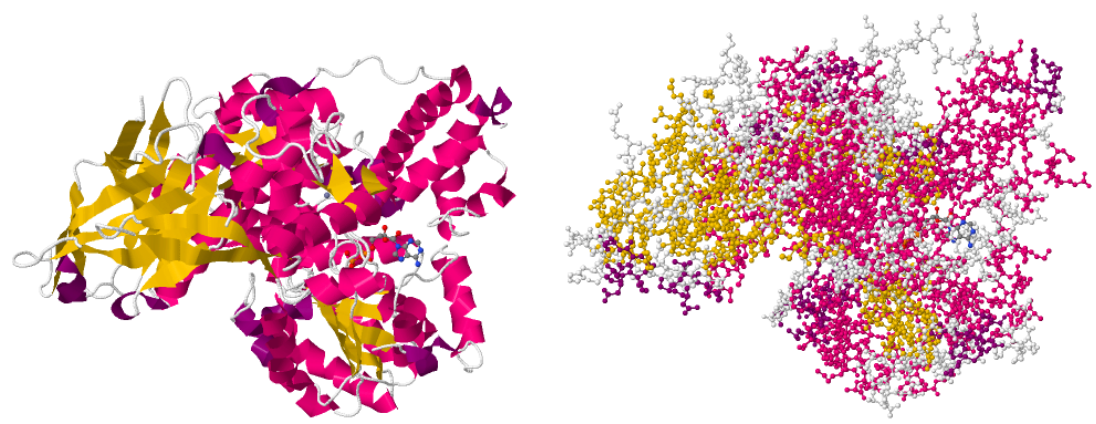

Figure 1: Tertiary structure of protein '4CDG’. Cartoon (left) and atomic (right) displays.

Although there are many different cancer types such that finding a coherent pattern representing their drivers is challenging, cancer manifests as tissue grows in an uncontrolled manner due to malfunction in the regular cell cycle process. Along with many other factors, it has been documented through experimentation that mutations in proto-oncogenes and in tumor suppressor genes and their regulatory mechanism play major roles in tumor growth. OGs refer to the genes that increase the number of cells while TSGs refer to the genes that control the cell growth process. Osborne et al [13] reviewed common OG and TSG malfunctions in human breast cancer. The OG/TSG detection improves the cancer identification performance as discussed in [14]. They have used genomic data and their variants from the cancer genomic atlas (TCGA), ICGC and COSMIC and have applied a random forest model integrating five statistical tests to detect cancer genes and specify them as likely OG and TSG. A question arises that how to classify OGs and TSGs only from their three-dimensional protein structures and the biochemical properties of the amino acids that forms the structure without extra statistical tests or other feature extraction modules? Prediction of the functional annotation of proteins is being improved by various methods such as prediction by sequence similarity [15, 16], evolutionary relations [17], genetic interactions [18], protein-protein interactions [19], protein structures and gene-ontology hierarchy [9, 20, 21].

In this paper, we propose a deep convolutional neural network (CNN) to classify TSGs and OGs based on their PDB structures and the biochemical properties. CNNs have shown high performances in visual feature extraction and classification [22, 23]. Additionally, CNNs provide hierarchical feature extraction modules which are robust against rotation, scale, and local translation. Thus, these types of visual extraction modules can be used to discover discriminative information of the PDB structures by mapping the biochemical properties annotated with 3-D atomic coordinates appearing on the outer surfaces to visual feature maps.

\section{TSG and OG Dataset Preparation}

\subsection{Protein Structure}

The tertiary protein structure is determined by three-dimensional geometric shape with a single polypeptide backbone. It contains a variety of bonding interactions between the side chains on the amino acids. Figure 1 shows a protein structure in which the colors exhibit its secondary structure. In this paper, we concentrate on the protein's atomic coordinates appearing on the outer surfaces $(\mathrm{x}, \mathrm{y}, \mathrm{z})$ and their associated biochemical properties.

\subsection{Biochemical Features}

Experimentally annotated genes that are related to cancer are downloaded from COSMIC (https: //cancer.sanger.ac.uk/census) V82 for human (GRCh38). For the purpose of this machine learning experiment in identifying the genes role in cancer from their 3D structure, we have focused on tumor suppressor genes and oncogenes. The recent version of the COSMIC annotated gene lists has 137 TSG and 78 oncogenes. These gene sets are combined together and are clustered with DAVID Bioinformatics Resources 6.8 [24] using only direct annotation from gene ontology (http://www.geneontology.org) and other functional categories provided by the tool. The gene ontology [25] provides a structured vocabulary to annotate genes and their products by providing 


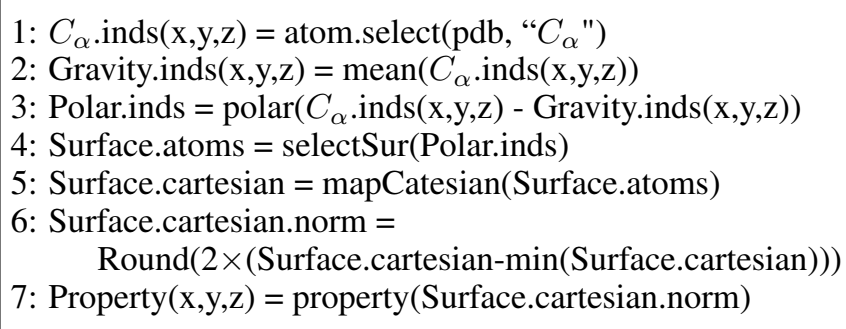

Figure 2: Pseudo-code for PDB feature extraction.

three orthogonal ontologies: biological process (BP), cellular components (CC), and molecular function (MF), each of which is modeled as a directed acyclic graph. As expected, the TSGs and the OGs are clustered into non-overlapping, separate clusters. This confirms that they are appropriate candidates for separate functional predictive models.

The Ensembl ids of OGs and TSGs are mapped to the PDB ids by using the UniProt web tool [26] (http://www.uniprot.org/uploadlists). The PDB files were downloaded from the protein data bank website (https://www.rcsb.org/pdb/download/download.do\#Structures). The PDB format contains a standard format for macromolecular structure data obtained by X-ray diffraction and NMR studies [27].

To interpret and distinguish these genes, we provide a feature extraction module to represent morphological characteristics of their tertiary structure. The feature extraction module has two folds: 1 ) identifying the surface $C_{\alpha}$ atoms; 2) extracting the surface atoms' properties. The pseudo code of feature extraction is shown in Figure 2

\subsubsection{Surface $C_{\alpha}$ Identifying}

For each PDB file, the surface $C_{\alpha}$ atoms are chosen. To find the surface atoms, the Cartesian coordinates are changed to polar coordinates from the centroid and then, with 1 degree resolution, the highest radius atom is selected as the surface atom (Figure 2 lines 1 through 5). Finally, the surface indices $\langle x, y, z\rangle$ are converted to decimal numbers starting from $\langle 0,0,0\rangle$ (Figure 2 line 6).

\subsubsection{Atom Properties}

The PDB files provide information for amino-acids placed in the $C_{\alpha}$ coordinates. In our model, each PDB file is represented by sixteen features along with their 3-D coordinates. These 16 features are extracted according to the corresponding amino-acids collected from http://www proteinstructures.com/Structure/Structure/amino-acids.html. The sixteen features that are contributed to the 20 amino-acids are shown in Table 1 . These features are used in the "property" function as shown in Figure 2 line 7. Note: the feature values of Pka-NH2 and P-Ka-COOH are normalized in the range $[0,1]$.

\section{Deep Learning Model}

In this section, we first explain the data processing steps required for preparing the feature maps fed into the CNN; and then, the network architecture is explained.

\subsection{Input Feature Maps}

As mentioned earlier, each PDB file is represented by 16 features associated with the atomic coordinates $\langle x, y, z\rangle$. To covert the three dimensional feature space to the feature maps (2-D), we generate three independent feature sets associated with three atomic projections on $\langle x, y\rangle,\langle y, z\rangle$, and $\langle x, z\rangle$ feature spaces. Therefore, each PDB file can be converted to three perpendicular 2-D feature spaces. In the next step, each projection is converted to 16 feature maps corresponding to the sixteen feature values computed in the previous section. 
bioRxiv preprint doi: https://doi.org/10.1101/177378; this version posted October 22, 2017. The copyright holder for this preprint (which was not certified by peer review) is the author/funder, who has granted bioRxiv a license to display the preprint in perpetuity. It is made available under aCC-BY 4.0 International license.

Table 1: Amino-acids and their associated properties.

\begin{tabular}{|c|c|c|c|c|c|c|c|c|c|c|c|c|c|c|c|c|c|c|c|c|c|c|}
\hline \multicolumn{3}{|c|}{ Amino acid short form } & $\stackrel{\text { 인 }}{\frac{4}{4}}$ & $\stackrel{n}{3}$ & 量 & 근 & $\frac{5}{\mathrm{~V}}$ & 号 & $\stackrel{\underline{x}}{\underline{x}}$ & 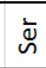 & $\stackrel{\grave{F}}{\vdash}$ & $\stackrel{亡}{\rightleftarrows}$ & $\stackrel{n}{u}$ & $\sum^{ \pm}$ & 은 & $\frac{\pi}{4}$ & $\stackrel{\varrho}{=}$ & $\stackrel{\varrho}{3}$ & $\stackrel{\frac{\Perp}{2}}{a}$ & $\bar{\Gamma}$ & $\stackrel{0}{2}$ & जे \\
\hline \multicolumn{3}{|c|}{ Amino acid code } & $\simeq$ & 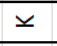 & 0 & س & $\sigma$ & $z$ & $I$ & $n$ & $\vdash$ & $>$ & $u$ & $\Sigma$ & 3 & $\varangle$ & - & 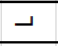 & 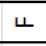 & $>$ & $a$ & $\circlearrowleft$ \\
\hline \multirow{16}{*}{ 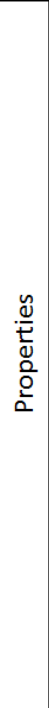 } & \multirow{3}{*}{ إِ } & charged & 1 & 1 & 1 & 1 & 0 & 0 & 0 & 0 & 0 & 0 & 0 & 0 & 0 & 0 & 0 & 0 & 0 & 0 & 0 & 0 \\
\hline & & Polar & 0 & 0 & 0 & 0 & 1 & 1 & 1 & 1 & 1 & 1 & 1 & 1 & 1 & 0 & 0 & 0 & 0 & 0 & 0 & 0 \\
\hline & & Hydrophobic & 0 & 0 & 0 & 0 & 0 & 0 & 0 & 0 & 0 & 0 & 0 & 0 & 0 & 1 & 1 & 1 & 1 & 1 & 1 & 1 \\
\hline & \multirow{13}{*}{ 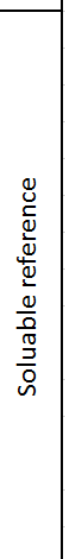 } & Hydrophobic & 0 & 0 & 0 & 0 & 0 & 0 & 0 & 0 & 0 & 1 & 0 & 0 & 1 & 1 & 1 & 1 & 1 & 1 & 1 & 1 \\
\hline & & Moderate & 0 & 0 & 0 & 0 & 0 & 0 & 1 & 0 & 0 & 0 & 1 & 1 & 0 & 0 & 0 & 0 & 0 & 0 & 0 & 0 \\
\hline & & Hydrophillic & 1 & 1 & 1 & 1 & 1 & 1 & 0 & 1 & 1 & 0 & 0 & 0 & 0 & 0 & 0 & 0 & 0 & 0 & 0 & 0 \\
\hline & & polar & 0 & 0 & 0 & 0 & 1 & 1 & 0 & 1 & 1 & 0 & 1 & 1 & 0 & 0 & 0 & 0 & 0 & 0 & 0 & 0 \\
\hline & & Aromatic & 0 & 0 & 0 & 0 & 0 & 0 & 0 & 0 & 0 & 1 & 0 & 0 & 1 & 0 & 0 & 0 & 1 & 0 & 0 & 0 \\
\hline & & Aliphatic & 0 & 0 & 0 & 0 & 0 & 0 & 0 & 0 & 0 & 0 & 0 & 0 & 0 & 1 & 1 & 1 & 0 & 1 & 0 & 0 \\
\hline & & Acidic & 0 & 0 & 1 & 1 & 0 & 0 & 0 & 0 & 0 & 0 & 0 & 0 & 0 & 0 & 0 & 0 & 0 & 0 & 0 & 0 \\
\hline & & Basic & 1 & 1 & 0 & 0 & 0 & 0 & 1 & 0 & 0 & 0 & 0 & 0 & 0 & 0 & 0 & 0 & 0 & 0 & 0 & 0 \\
\hline & & $\begin{array}{l}\text { Negative } \\
\text { charge }\end{array}$ & 0 & 0 & 1 & 1 & 0 & 0 & 0 & 0 & 0 & 0 & 0 & 0 & 0 & 0 & 0 & 0 & 0 & 0 & 0 & 0 \\
\hline & & Neutral & 0 & 0 & 0 & 0 & 1 & 1 & 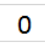 & 1 & 1 & 1 & 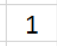 & 1 & 1 & 1 & 1 & 1 & 1 & 1 & 1 & 1 \\
\hline & & $\begin{array}{l}\text { Positive } \\
\text { charge }\end{array}$ & 1 & 1 & 0 & 0 & 0 & 0 & 1 & 0 & 0 & 0 & 0 & 0 & 0 & 0 & 0 & 0 & 0 & 0 & 0 & 0 \\
\hline & & Pka_NH2 & 9 & 10 & 9.6 & 10 & 9 & 9 & 9 & 9 & 9 & 9 & 11 & 9.2 & 9.4 & 9.9 & 10 & 9.6 & 9 & 9.7 & 11 & 9.6 \\
\hline & & P_ka_COOH & 2 & 9 & 1.9 & 2 & 2 & 2 & 2 & 2 & 2 & 2 & 1.7 & 2.3 & 2.4 & 2.4 & 2 & 2.4 & 3 & 2.3 & 2 & 2.3 \\
\hline
\end{tabular}

Table 2: The CNN's parameters.

\begin{tabular}{|l|l|l|l|}
\hline Parameter & Value & Parameter & Value \\
\hline$d_{1}$ & 32 & $s_{1}$ & 4 \\
\hline$d_{2}$ & 32 & $s_{2}$ & 2 \\
\hline$d_{3}$ & 64 & $s_{3}$ & 2 \\
\hline$d_{4}$ & 64 & $s_{4}$ & 2 \\
\hline$p$ & $3-9$ & $\gamma$ & 2 \\
\hline$h_{1}$ & 100 & $h_{2}$ & 50 \\
\hline
\end{tabular}

This approach converts a 3-D structure to three feature map sets with dimensions of $200 \times 200 \times 16$ pixels $(16$ feature maps of $200 \times 200$ ). Processing the projections is much faster than processing the 3-D structures while not losing information considerably due to the PDB's sparse structure. Furthermore, each feature map set of a projection denotes specific features of the protein while preserving its spatial information. Figure 3 shows the feature maps of one of the three projections $(<x, y>)$ for a TSG.

\subsection{CNN Architecture}

The deep learning model, in this study, develops a parallel CNN with three branches followed by a multi-layer fully connected neural network. Figure 4 shows this deep CNN's architecture. The model consists of four convolution and pooling layers and three fully connected layers including the final classifier. The convolution kernel size $(p)$, pooling strides $\left(s_{i}\right)$, number of hidden neurons $\left(h_{1}, h_{2}\right)$, convolution padding $(\gamma)$, and the number of generated feature maps $\left(d_{i}\right)$ are shown in Table 2 . These parameters have been set up after a number of control experiments and initial evaluations.

As shown in Figure 4 the $\mathrm{CNN}$ receives three $200 \times 200 \times 16$ feature maps in parallel and performs a binary (TSG/OG) classification. Each layer is equipped by the rectified linear unit (ReLU) activation function. We used 30\% dropout in the fully connected layers to control probable over training. More details about the network's training process is discussed in the next section (Experiments). The features utilized for generating the feature maps are shown on the left side of the network (Figure 4). The convolution/pooling layers extract $108 \times 64=6912$ visual features. The number of trainable parameters are shown on the right side of the network. If we consider $p=5$, we will have 888,250 trainable parameters. 


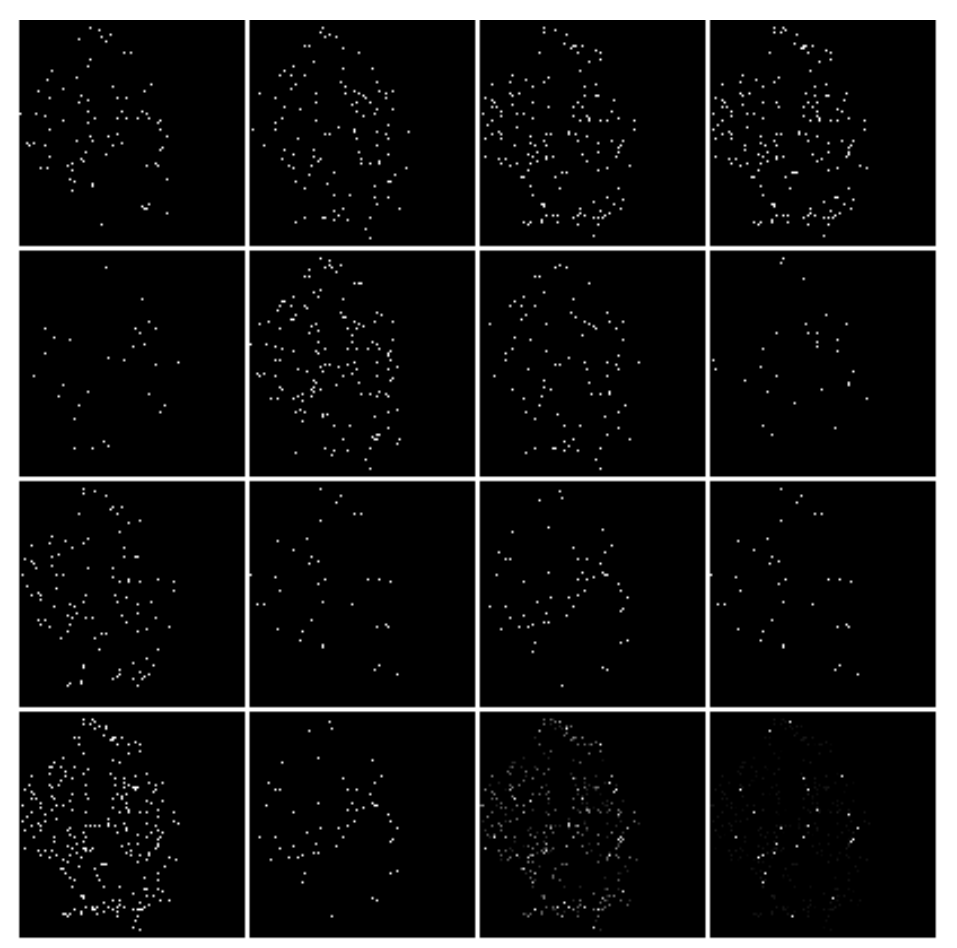

Figure 3: Sixteen feature maps obtained from the $<x, y>$ projection of protein '4CDG' (a TSG). Each map illustrates one of the biological features calculated in the previous section. The row-wise map order is analogous to the order of features shown in Fig.4

\section{Experiments and Results}

The proposed model is evaluated on the dataset that we collected in Section 2 . The dataset consists of 2379 PDB files (1191 TSG and 1188 OG) that is converted to 7137 feature maps with 16 channels. The 2379 feature map sets, each representing one particular protein structure, are randomly divided into separate training and testing sets with 2029 training and 350 testing samples. This dataset division method is repeated three times using different random seeds. Finally, the model is trained and evaluated over 100 iterations.

We implemented the model using the Torch library [28] available via: https://github.com/ tavanaei/Cancer-Suppressor-Gene-Deep-Learning.

\subsection{Results}

We ran four evaluations on the CNNs with different convolution kernel sizes $(p=\{3,5,7,9\})$ to find a proper patch size for extracting the feature maps' visual features. Figure 5 illustrates the CNNs' accuracy rates over 100 training iterations. It is shown that the $3 \times 3$ patch is not able to discover the protein maps' features well. The best accuracy belongs to the networks with convolution kernels with $p=\{7,9\}$ patch sizes. Table 3 shows the detailed performance measures of the proposed model. The best performed model reported accuracy rate of $82.57 \%$ and area under the ROC curve (AUROC) of 0.89 . The test sets used to generate Figure 5 and Table 3 are slightly different (fewer samples were used for the plot).

To asses the model's convergence speed, Figure 6 shows the model's performance with respect to different learning rates. The models trained by the learning rates $\mu>0.03$ reach accuracy rates higher than $75 \%$ after 20 iterations. The best performing models were trained using learning rates of 0.05 and 0.06 . Table 4 also shows high performances for these learning rates while it is evaluated on a slightly different test set (same as Table 3 ). 
bioRxiv preprint doi: https://doi.org/10.1101/177378; this version posted October 22, 2017. The copyright holder for this preprint (which was not certified by peer review) is the author/funder, who has granted bioRxiv a license to display the preprint in perpetuity. It is made available under aCC-BY 4.0 International license.

4.1 Results 4 EXPERIMENTS AND RESULTS

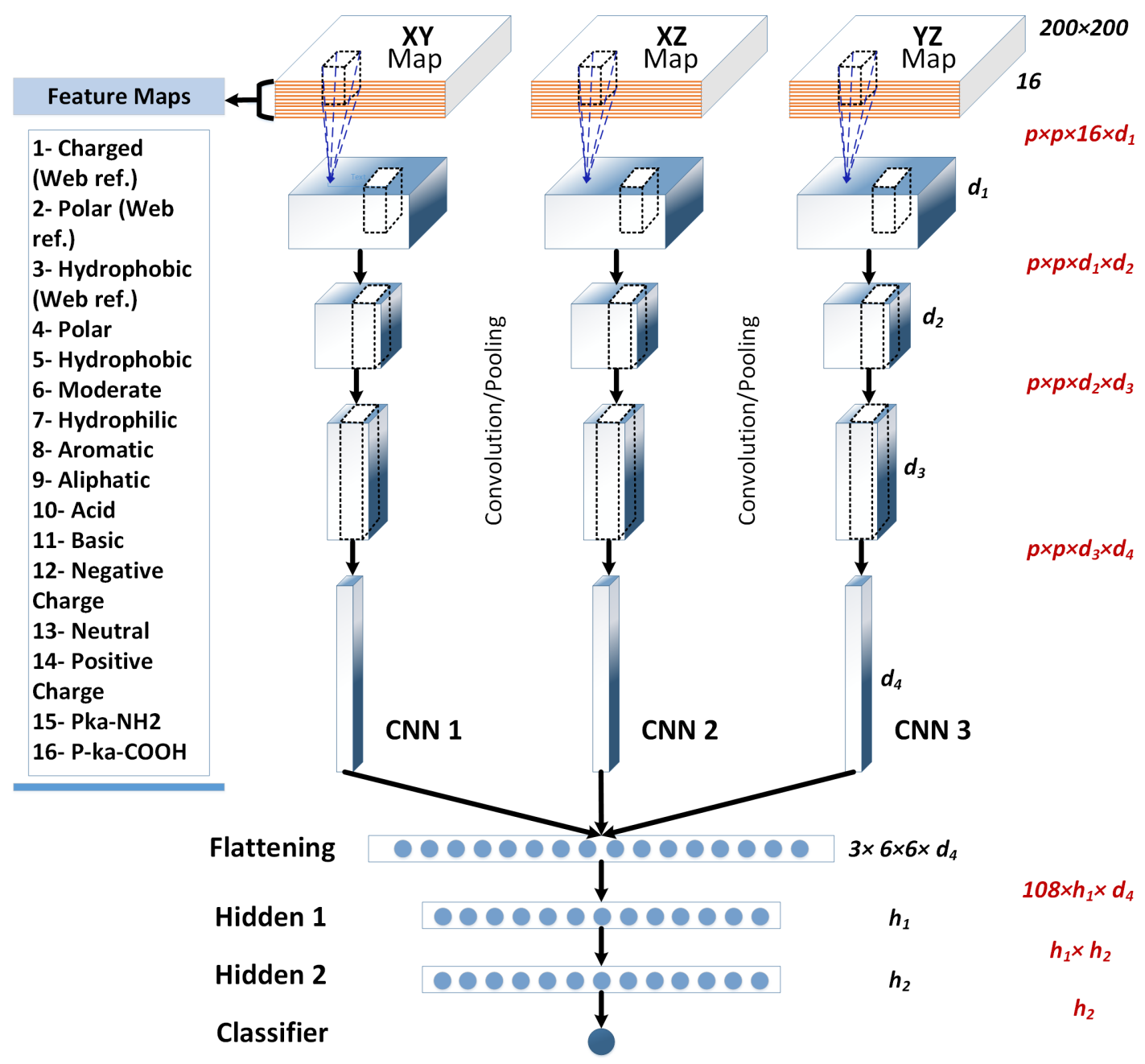

Figure 4: The deep CNN's architecture for OG/TSG prediction. For simplicity, the convolution and pooling layers are shown as one module. The red expressions calculate the number of trainable parameters for each layer.

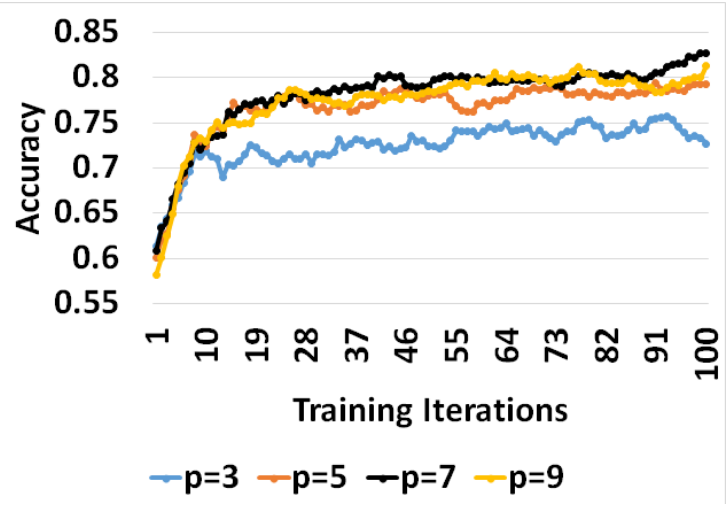

Figure 5: Accuracy of the networks with $p=\{3,5,7,9\}$ convolution patch sizes over training. $\mu=0.05$ 
bioRxiv preprint doi: https://doi.org/10.1101/177378; this version posted October $22,2017$. The copyright holder for this preprint (which was not certified by peer review) is the author/funder, who has granted bioRxiv a license to display the preprint in perpetuity. It is made available under aCC-BY 4.0 International license.

Table 3: Performance of the CNNs in terms of accuracy, precision, recall, and area under ROC (AUROC). $\mu=0.05$.

\begin{tabular}{|l|c|c|c|}
\hline \multicolumn{1}{|c|}{$\boldsymbol{p}$} & $\mathbf{5}$ & $\mathbf{7}$ & $\mathbf{9}$ \\
\hline Accuracy (\%) & 81.71 & $\mathbf{8 2 . 5 7}$ & 79.43 \\
\hline Recall (\%) & 85.80 & 81.87 & 80.70 \\
\hline Precision (\%) & 78.38 & 82.35 & 77.97 \\
\hline AUROC & 0.881 & $\mathbf{0 . 8 8 7}$ & 0.869 \\
\hline
\end{tabular}

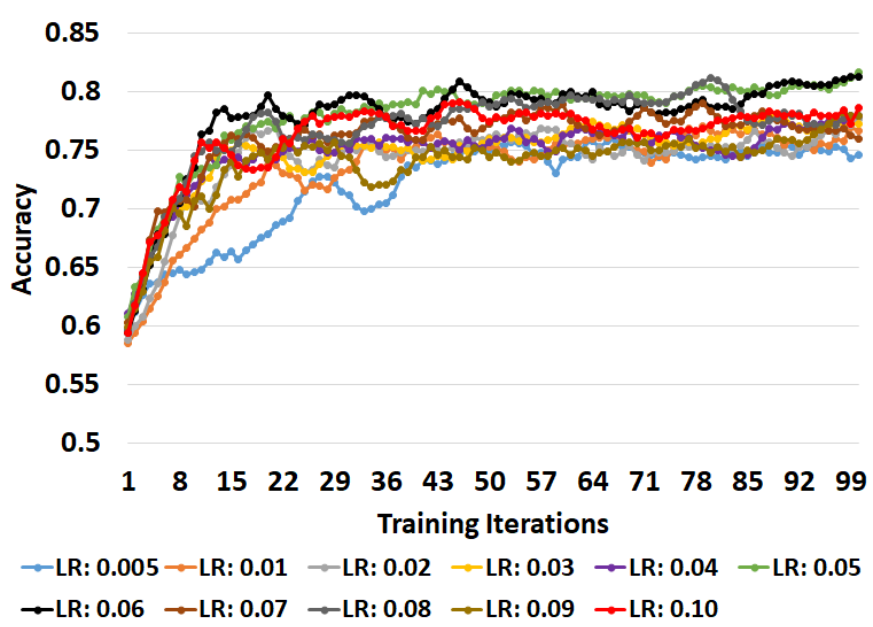

Figure 6: Accuracy of the CNN over training versus learning rate. $\mu=0.05$ and $p=7$.

\subsection{Summary and Discussion}

The raw input data are obtained from the 3D structures of proto-oncogenes and cancer suppression genes. The function and the biological processes of a protein are dependent on the outer surface configuration and their biochemical properties. We have developed an algorithm to identify atoms located on the outer surface and have used selected sixteen properties of the amino acids as shown in Table 1. The 3D configuration of the outer surface is mapped onto three orthogonal planes. Each property becomes a channel in the feature map of the CNN as illustrated in Figure 4 The proposed CNN model with the 16 channel feature map achieved a remarkable performance of $82.6 \%$ accuracy rate and 0.89 AUROC. The model becomes very useful in annotating uncharacterized PDB structures into either the TSG or the OG structures.

Furthermore, this performance of our model compares favorably with the statistical methods studied by [14] on pan-cancer genome sequencing data [29] which consists of very rich genomic information. Event though the dataset we used for evaluating our model is different from their dataset. Table 5

Table 4: Performance of the CNNs versus learning rate. Convolution patch size $p=7$.

\begin{tabular}{|l|l|l|l|l|}
\hline Learning Rate & Accuracy & Recall & Precision & AUROC \\
\hline $\mathbf{0 . 0 0 5}$ & 78.29 & 86.98 & 73.13 & 0.880 \\
\hline $\mathbf{0 . 0 1 0}$ & 77.71 & 81.66 & 74.59 & 0.871 \\
\hline $\mathbf{0 . 0 2 0}$ & 80.00 & 85.80 & 75.92 & 0.876 \\
\hline $\mathbf{0 . 0 3 0}$ & 79.14 & 83.43 & 75.81 & 0.878 \\
\hline $\mathbf{0 . 0 4 0}$ & 80.57 & 80.00 & 80.00 & 0.872 \\
\hline $\mathbf{0 . 0 5 0}$ & $\mathbf{8 2 . 5 7}$ & 81.87 & 82.35 & $\mathbf{0 . 8 8 7}$ \\
\hline $\mathbf{0 . 0 6 0}$ & 82.00 & 85.12 & 79.01 & $\mathbf{0 . 8 8 3}$ \\
\hline $\mathbf{0 . 0 7 0}$ & 79.14 & 79.29 & 77.91 & 0.877 \\
\hline $\mathbf{0 . 0 8 0}$ & 82.00 & 82.84 & 80.46 & 0.880 \\
\hline $\mathbf{0 . 0 9 0}$ & 78.57 & 85.21 & 74.23 & 0.871 \\
\hline $\mathbf{0 . 1 0 0}$ & 80.00 & 81.66 & 77.97 & 0.874 \\
\hline
\end{tabular}


Table 5: AUROC of OG/TSG identification using the statistical methods reported by [14] and our model.

\begin{tabular}{|l|l|l|}
\hline \multicolumn{1}{|c|}{ Method } & \multicolumn{1}{c|}{ Description } & AUROC \\
\hline Truncation Rate & Rate of truncating events* & $\mathbf{0 . 9 2 2}$ \\
\hline Unaffected Residues & $\begin{array}{l}\text { Intra-gene mutation } \\
\text { clustering/recurrence* }\end{array}$ & 0.479 \\
\hline VEST Mean & Functional impact bias* & 0.71 \\
\hline Patient Distribution & Bias in patient labels* & 0.556 \\
\hline 'Cancer Type Distribution & Cancer type bias* & 0.612 \\
\hline Oncodrive-fm & $\begin{array}{l}\text { Gonzalez-Perez and } \\
\text { Lopez-Bigas [30] }\end{array}$ & 0.725 \\
\hline Oncodrive-CLUST & Tamborero et al., [31] & 0.597 \\
\hline Random Forest & $\begin{array}{l}\text { Ensemble on the } \\
\text { first 5 methods }(*)\end{array}$ & $\mathbf{0 . 9 2 4}$ \\
\hline Our Model & $\begin{array}{l}\text { DCNN applied to } \\
\text { the PDB structure }\end{array}$ & $\mathbf{0 . 8 8 7}$ \\
\hline
\end{tabular}

compares the AUROC value reported in our study with the AUROC values reported by state-of-the-art statistical methods for OG versus TSG identification. Our model outperforms the six out of eight methods and is close to the best AUROC.

\section{Conclusion}

A deep learning approach was proposed in this paper to classify the cancer genes: proto-oncogenes and tumor suppressor genes. By having a model that confidently identifies proto-oncogene or cancer suppressor genes from the structure and the biochemical properties of amino acids, we are providing a general methodology that will lead to a new tool to discover a new set of cancer suppressor genes or proto-oncogenes that may not have been identified in the literature of having such functionality. By activating such dormant cancer suppression gene through drugs, we improve the chances of controlling tumor growth. Of course, the identified potential cancer suppression genes have to be verified through testing with rat or mouse model which resemble human gene content.

This investigation was established in two steps: 1) Protein feature extraction from the PDB tertiary structure; 2) modeling the gene patterns using a parallel deep convolutional neural network (CNN). The proposed DCNN preserves the spatial information of the tertiary structure while modeling the protein structure/features via three parallel, independent visual feature extraction modules. Finally, the fully connected neural network of the DCNN classifies the combined visual features.

The experimental results showed high performance of $82.57 \%$ and 0.887 accuracy rate and area under the ROC curve, respectively. The initial success of our model warrants our future study to apply the same deep learning approach on new datasets for predicting different cancer types to identify the cancer drivers.

\section{References}

[1] K. Kourou, T. P. Exarchos, K. P. Exarchos, M. V. Karamouzis, and D. I. Fotiadis, "Machine learning applications in cancer prognosis and prediction," Computational and structural biotechnology journal, vol. 13, pp. 8-17, 2015.

[2] S. Vural, X. Wang, and C. Guda, "Classification of breast cancer patients using somatic mutation profiles and machine learning approaches," BMC systems biology, vol. 10, no. 3, p. 62, 2016.

[3] C. Sotiriou, S.-Y. Neo, L. M. McShane, E. L. Korn, P. M. Long, A. Jazaeri, P. Martiat, S. B. Fox, A. L. Harris, and E. T. Liu, "Breast cancer classification and prognosis based on gene expression profiles from a population-based study," Proceedings of the National Academy of Sciences, vol. 100, no. 18, pp. 10393-10398, 2003.

[4] J. A. Cruz and D. S. Wishart, "Applications of machine learning in cancer prediction and prognosis," Cancer informatics, vol. 2, p. 59, 2006. 
[5] Z. Cai, D. Xu, Q. Zhang, J. Zhang, S.-M. Ngai, and J. Shao, “Classification of lung cancer using ensemble-based feature selection and machine learning methods," Molecular BioSystems, vol. 11, no. 3, pp. 791-800, 2015.

[6] Y. LeCun, Y. Bengio, and G. Hinton, “Deep learning,” Nature, vol. 521, no. 7553, pp. 436-444, 2015.

[7] J. Schmidhuber, "Deep learning in neural networks: An overview," Neural networks, vol. 61, pp. 85-117, 2015.

[8] B. Alipanahi, A. Delong, M. T. Weirauch, and B. J. Frey, "Predicting the sequence specificities of DNA- and RNA-binding proteins by deep learning," Nature biotechnology, vol. 33, no. 8, pp. $831-838,2015$.

[9] A. Tavanaei, A. S. Maida, A. Kaniymattam, and R. Loganantharaj, "Towards recognition of protein function based on its structure using deep convolutional networks," in Bioinformatics and Biomedicine (BIBM), 2016 IEEE International Conference on. IEEE, 2016, pp. 145-149.

[10] T. Jo, J. Hou, J. Eickholt, and J. Cheng, "Improving protein fold recognition by deep learning networks," Scientific reports, vol. 5, p. srep17573, 2015.

[11] R. Fakoor, F. Ladhak, A. Nazi, and M. Huber, "Using deep learning to enhance cancer diagnosis and classification," in Proceedings of the International Conference on Machine Learning, 2013.

[12] D. Wang, A. Khosla, R. Gargeya, H. Irshad, and A. H. Beck, "Deep learning for identifying metastatic breast cancer," arXiv preprint arXiv:1606.05718, 2016.

[13] C. Osborne, P. Wilson, and D. Tripathy, "Oncogenes and tumor suppressor genes in breast cancer: potential diagnostic and therapeutic applications," The oncologist, vol. 9, no. 4, pp. 361-377, 2004.

[14] R. D. Kumar, A. C. Searleman, S. J. Swamidass, O. L. Griffith, and R. Bose, "Statistically identifying tumor suppressors and oncogenes from pan-cancer genome-sequencing data," Bioinformatics, vol. 31, no. 22, pp. 3561-3568, 2015.

[15] M. Kulmanov, M. A. Khan, and R. Hoehndorf, "Deepgo: Predicting protein functions from sequence and interactions using a deep ontology-aware classifier," arXiv preprint arXiv:1705.05919, 2017.

[16] Y. Loewenstein, D. Raimondo, O. C. Redfern, J. Watson, D. Frishman, M. Linial, C. Orengo, J. Thornton, and A. Tramontano, "Protein function annotation by homology-based inference," Genome biology, vol. 10, no. 2, p. 207, 2009.

[17] P. Gaudet, M. S. Livstone, S. E. Lewis, and P. D. Thomas, "Phylogenetic-based propagation of functional annotations within the gene ontology consortium," Briefings in bioinformatics, vol. 12, no. 5, pp. 449-462, 2011.

[18] M. Costanzo, B. VanderSluis, E. N. Koch, A. Baryshnikova, C. Pons, G. Tan, W. Wang, M. Usaj, J. Hanchard, S. D. Lee et al., "A global genetic interaction network maps a wiring diagram of cellular function,” Science, vol. 353, no. 6306, p. aaf1420, 2016.

[19] R. Sharan, I. Ulitsky, and R. Shamir, "Network-based prediction of protein function," Molecular systems biology, vol. 3, no. 1, p. 88, 2007.

[20] J. Konc, M. Hodošček, M. Ogrizek, J. T. Konc, and D. Janežič, "Structure-based function prediction of uncharacterized protein using binding sites comparison," PLoS computational biology, vol. 9, no. 11, p. e1003341, 2013.

[21] A. Sokolov and A. Ben-Hur, "Hierarchical classification of gene ontology terms using the GOstruct method," Journal of bioinformatics and computational biology, vol. 8, no. 02, pp. 357-376, 2010.

[22] A. Krizhevsky, I. Sutskever, and G. E. Hinton, "Imagenet classification with deep convolutional neural networks," in Advances in neural information processing systems, 2012, pp. 1097-1105. 
[23] W. Rawat and Z. Wang, "Deep convolutional neural networks for image classification: A comprehensive review," Neural Computation, 2017.

[24] D. W. Huang, B. T. Sherman, Q. Tan, J. R. Collins, W. G. Alvord, J. Roayaei, R. Stephens, M. W. Baseler, H. C. Lane, and R. A. Lempicki, "The DAVID gene functional classification tool: a novel biological module-centric algorithm to functionally analyze large gene lists," Genome biology, vol. 8, no. 9, p. R183, 2007.

[25] M. Ashburner, C. A. Ball, J. A. Blake, D. Botstein, H. Butler, J. M. Cherry, A. P. Davis, K. Dolinski, S. S. Dwight, J. T. Eppig et al., "Gene ontology: tool for the unification of biology," Nature genetics, vol. 25, no. 1, p. 25, 2000.

[26] U. Consortium et al., "Uniprot: a hub for protein information," Nucleic acids research, p. gku989, 2014.

[27] H. M. Berman, J. Westbrook, Z. Feng, G. Gilliland, T. N. Bhat, H. Weissig, I. N. Shindyalov, and P. E. Bourne, "The protein data bank, 1999-," in International Tables for Crystallography Volume F: Crystallography of biological macromolecules. Springer, 2006, pp. 675-684.

[28] R. Collobert, K. Kavukcuoglu, and C. Farabet, "Torch7: A matlab-like environment for machine learning," in BigLearn, NIPS Workshop, 2011.

[29] J. N. Weinstein, E. A. Collisson, G. B. Mills, K. R. M. Shaw, B. A. Ozenberger, K. Ellrott, I. Shmulevich, C. Sander, J. M. Stuart, C. G. A. R. Network et al., "The cancer genome atlas pan-cancer analysis project," Nature genetics, vol. 45, no. 10, pp. 1113-1120, 2013.

[30] A. Gonzalez-Perez and N. Lopez-Bigas, "Functional impact bias reveals cancer drivers," Nucleic acids research, vol. 40, no. 21, pp. e169-e169, 2012.

[31] D. Tamborero, A. Gonzalez-Perez, and N. Lopez-Bigas, “OncodriveCLUST: exploiting the positional clustering of somatic mutations to identify cancer genes," Bioinformatics, vol. 29, no. 18, pp. 2238-2244, 2013. 